\title{
PHOTOCATALYTIC OXIDATION OF AROMATIC ALCOHOLS TO ALDEHYDES IN AQUEOUS SUSPENSION OF HOME PREPARED TITANIUM DIOXIDE 1. SELECTIVITY ENHANCEMENT BY ALIPHATIC ALCOHOLS
}

Vincenzo Augugliaro, ${ }^{a^{*}}$ Horst Kisch, ${ }^{\mathrm{b}}$ Vittorio Loddo, ${ }^{\mathrm{a}}$ María José López-Muñoz, Carlos Márquez-Álvarez, ${ }^{\mathrm{d}}$ Giovanni Palmisano, ${ }^{\mathrm{a}}$ Leonardo Palmisano, ${ }^{\text {a* }}$ Francesco Parrino, ${ }^{\mathrm{b}}$ Sedat Yurdakal ${ }^{\mathrm{a}, \mathrm{e}}$

a "Schiavello-Grillone" Photocatalysis Group, Dipartimento di Ingegneria Chimica dei Processi e dei Materiali, Università degli Studi di Palermo, Viale delle Scienze, 90128 Palermo, Italy

${ }^{\mathrm{b}}$ Institute of Inorganic Chemistry, University of Erlangen-Nürnberg, Egerlandstrasse 1, D-91058 Erlangen, Germany

${ }^{c}$ Department of Chemical and Environmental Technology, ESCET, Rey Juan Carlos University, C/Tulipán s/n, 28933 Móstoles, Madrid, Spain

d Instituto de Catálisis y Petroleoquímica, CSIC, C/Marie Curie 2, 28049 Cantoblanco, Madrid, Spain

${ }^{\text {e } K i m y a ~ B o ̈ l u ̈ m u ̈, ~ F e n ~ F a k u ̈ l t e s i, ~ A n a d o l u ~ U ̈ n i v e r s i t e s i, ~ Y u n u s ~ E m r e ~ K a m p u ̈ s u ̈, ~} 26470$ Eskişehir, Turkey

Dedicated to the friendship between Pablo Neruda and Nazım Hikmet

${ }^{*}$ Corresponding authors

E-mail: augugliaro@dicpm.unipa.it,palmisano@dicpm.unipa.it

KEYWORDS: photocatalysis, $\mathrm{TiO}_{2}$, aromatic alcohols, aliphatic alcohol, selective oxidation 


\begin{abstract}
Oxygenated aqueous suspensions of home-prepared (HP) and commercial $\mathrm{TiO}_{2}$ catalysts were used in a batch photoreactor for carrying out the oxidation of benzyl alcohol (BA) and 4methoxybenzyl alcohol (MBA) under different operative conditions. HP catalysts were synthesized from $\mathrm{TiCl}_{4}$ and underwent a hydrolysis treatment of different times under mild conditions. The textural characterisation of catalysts was carried out with XRD, SEM observations, BET surface area and porosity measurements. For both alcohols the main oxidation products were the corresponding aromatic aldehydes and $\mathrm{CO}_{2}$. The $\mathrm{HP}$ catalysts exhibited selectivity values towards the aldehyde production up to $28 \%$ (BA conversion: $50 \%$ ) and $41 \%$ (MBA conversion: $65 \%$ ), about four times higher than those of commercial $\mathrm{TiO}_{2}$. The addition of an aliphatic alcohol (methanol, ethanol, 2-propanol or tert-butanol) in small amounts with respect to water decreased the overall oxidation rate of aromatic alcohols but enhanced the selectivity for aldehyde formation up to 1.5 times. The reactivity results suggest that: (i) the aromatic alcohol molecules interact with the $\mathrm{TiO}_{2}$ surface in different ways that eventually determine two parallel reaction pathways (partial oxidation or mineralization); and (ii) the aliphatic alcohols preferentially compete with aromatic alcohols for the mineralizing pathway.
\end{abstract}




\section{INTRODUCTION}

The increasingly stringent environmental limitations for chemical processes are driving scientists all over the world to investigate the replacement of contaminant processes with new ones, preferably taking place in atoxic (or even no) solvents. A new branch of organic chemistry is being born in which all reactions develop in water, often with high reaction rates even for insoluble reagents [1-5].

The selective oxidation of aliphatic and aromatic alcohols to their corresponding carbonyl compounds (aldehydes, carboxylic acids and ketones) is an essential reaction frequently encountered in the synthesis of fine chemicals [6]. Traditional methods for performing such partial oxidations involve the use of heavy metal compounds, as manganates and chromates, or organic oxidants often in chlorinated solvents. Generally such oxidizing reagents are hazardous and toxic and show low selectivities so that, when used in stoichiometric amounts, generate large quantities of waste whose treatment increases the cost of the overall process.

The development of processes with clean oxidants and stable catalysts is of great economical and environmental concern [7]. A homogeneous catalytic system effective for aerobic oxidation of alcohols to carbonyl compounds is a water-soluble palladium(II) bathophenanthroline disulfonate complex [8]. This method enables to oxidise a wide range of both primary and secondary allylic, benzylic and aliphatic alcohols in high conversions and selectivities at 30 bar and $376 \mathrm{~K}$. The conversion is almost complete and the selectivity is in the $79-90 \%$ range depending on the used substrate; moreover no oxidation of di-substituted aromatic alcohols occurs. The catalytic oxidation of various alcohols, including primary aliphatic alcohols to aldehydes by $\mathrm{O}_{2}$ has been also performed with a $\mathrm{Au} / \mathrm{Pd}-\mathrm{TiO}_{2}$ catalyst [9], obtaining very high turnover frequencies. In the case of benzyl alcohol oxidation to benzaldehyde, a conversion of 74 $\%$ and a selectivity of $92 \%$ are reached and benzyl benzoate is the only detected by-product. The partial oxidation of benzyl alcohol has been also carried out with air under mild pressure and temperature over carbon supported platinum [10] and with hydrogen peroxide as oxygen donor using molybdenum- and tungsten-based catalytic systems and copper(II) complexes with tetraaza[14]annulene encapsulated within the nanocavities of Y-zeolites [11].

Heterogeneous photocatalysis is an advanced oxidation method nowadays mainly used for water and air remediation [12-15]. However, it has been also applied for performing selective 
oxidations [16-25] in the presence of polycrystalline $\mathrm{TiO}_{2}$ catalysts. Aqueous aerated suspensions are able to partially oxidise hydrocarbons to alcohols and carbonyl compounds upon artificial irradiation [20], and sunlight-induced functionalisation of some heterocyclic bases in the presence of water/acetonitrile leads to amido compounds [21]. Various primary and secondary alcohols have been selectively oxidised in a gas-phase photocatalytic reactor [22] using an immobilised catalyst; photooxidation of selected aryl alcohols to the corresponding aldehydes or ketones and acids has been carried out in acetonitrile solvent [23]. Palmisano et al. [24] have recently checked the feasibility of the photocatalytic oxidation of 4-methoxybenzyl alcohol (MBA) to 4-methoxybenzaldehyde (p-anisaldehyde, PAA) in aqueous suspensions of homeprepared $\mathrm{TiO}_{2}$ catalysts. The only by-products present were traces of 4-methoxybenzoic acid and open-ring products, $\mathrm{CO}_{2}$ being the other main oxidation product. They prepared nanostructured $\mathrm{TiO}_{2}$ of different crystallinity and observed that the selectivity to aldehyde is higher for less crystalline samples.

In the present investigation the photocatalytic oxidation of benzyl alcohol (BA) to benzaldehyde (BAD) and of MBA to PAA has been performed in organic-free water with $\mathrm{TiO}_{2}$ powders. Photoreactivity runs were carried out in aqueous suspensions of different homeprepared (HP) nanostructured specimens; for the sake of comparison also two commercial $\mathrm{TiO}_{2}$ catalysts, i.e. Degussa P25 and Merck, were included in this investigation. The textural characterization of HP and commercial catalysts was carried out with XRD, SEM observations, BET surface area and porosity measurements. The performances of catalysts were compared in terms of oxidation rate of aromatic alcohols and selectivity towards the aldehydes production.

With the aim of affecting the photoprocess selectivity, an aliphatic alcohol (methanol, ethanol, 2-propanol or tert-butanol) was added to the reacting system in small amounts with respect to water. These species are frequently used in heterogeneous photocatalytic systems due to their capability to work as strong hole traps [26-30]; here, however, the aliphatic alcohols are exploited to influence the performance of complex oxidation processes. 


\section{EXPERIMENTAL}

Photoreactivity runs were carried out in aqueous suspensions of different HP nanostructured $\mathrm{TiO}_{2}$ specimens; for the sake of comparison also two commercial $\mathrm{TiO}_{2}$ catalysts, i.e. Degussa P25 and Merck, were included in this investigation. The preparation method of HP catalysts is here summarized; the details are elsewhere reported [24]. The precursor solution was obtained by slowly adding $\mathrm{TiCl}_{4}$ to water under magnetic stirring that was prolonged for $12 \mathrm{~h}$ at room temperature, eventually obtaining a clear solution. This solution was heated at $373 \mathrm{~K}$ for $0.5,2,4,6$ and $8 \mathrm{~h}$, obtaining a white suspension at the end of each treatment. The suspension was then dried at $323 \mathrm{~K}$ in order to obtain the final powdered catalysts. The HP powders were washed with distilled water and centrifuged several times until the chloride ion concentration in the washing water reached a negligible value. Hereafter the HP catalysts are referred to as "HPx" in which the $\mathrm{x}$ figure indicates the boiling time of the precursor, expressed in hours.

XRD patterns of the powders were recorded by a diffractometer (Philips) using the $\mathrm{CuK}_{\alpha}$ radiation and a $2 \theta$ scan rate of $1.2 \% \mathrm{~min}$. SEM images were obtained using an ESEM microscope (XL30, Philips) operating at $25 \mathrm{kV}$ on samples suspended in water, sprayed on the stab and dried at room temperature, upon which a thin layer of gold had been evaporated. BET specific surface areas and porosities were measured by the multi-point BET method (Quantachrome 2000E).

A cylindrical batch photoreactor of Pyrex glass with immersed lamp was used for photocatalytic runs; details of the experimental set up are elsewhere reported [31]. The catalysts were used in $0.5 \mathrm{~L}$ aqueous suspensions well mixed by means of a magnetic stirrer and irradiated by a medium-pressure Hg lamp (Helios Italquarz, Italy). Before starting and during the runs, pure oxygen was continuously bubbled through the suspension. The dark equilibrium conditions were reached after 30 minutes mixing; at that time the lamp was switched on. Samples $\left(5 \mathrm{~cm}^{3}\right)$ for analysis where withdrawn every 1 or $2 \mathrm{~h}$; they were immediately filtered by means of a cellulose acetate filter (Millipore) with pore diameter of $0.45 \mu \mathrm{m}$. Average irradiance impinging onto the suspension was $10 \mathrm{~mW} \mathrm{~cm}^{-2}$; it was measured by using a radiometer (UVX, Digital) at $\lambda=360$ $\mathrm{nm}$. All the runs were duplicated; in the case the obtained results exceeded the $\pm 3 \%$ differences, the run was repeated until two runs were obtained in the above mentioned error range.

The quantitative determination and identification of the species present in the reacting suspension were performed by means of a HPLC (Beckman Coulter, System Gold 126 Solvent 
Module and 168 Diode Array Detector), equipped with a Luna $5 \mu$ Phenyl-Hexyl column (250 $\mathrm{mm}$ long $\times 2 \mathrm{~mm}$ i.d.), using standards (Sigma-Aldrich). The eluent consisted of: 17.5\% acetonitrile, $17.5 \%$ methanol, $65 \% 40 \mathrm{mM} \mathrm{KK_{2 }} \mathrm{PO}_{4}$ aqueous solution. The retention times and UV-spectra of the compounds were compared with those of authentic samples.

Aliphatic alcohol concentration was measured by using a gas chromatograph (GC-17A, Shimadzu) equipped with a methyl siloxane column $(30 \mathrm{~m} \times 320 \mu \mathrm{m} \times 0.25 \mu \mathrm{m}$, HP-1, HewlettPackard) and a flame ionization detector using He as carrier gas. For the methanol analysis a fixed quantity of liquid sample was injected into a gas-tight glass tube through a syringe, kept at constant temperature of $293 \mathrm{~K}$ for $20 \mathrm{~min}$. Upon reaching thermodynamic equilibrium, a $500 \mu \mathrm{L}$ sample of the headspace vapour was injected in the gas chromatograph. A multi-point calibration curve allowed to calculate the liquid phase concentrations. Total organic carbon (TOC) analyses were carried out by using a TOC analyzer (5000A, Shimadzu).

All the used chemicals (Sigma-Aldrich) had a purity $>99.0 \%$.

\section{RESULTS}

\subsection{Catalysts characterization}

All the information obtained by the textural characterisation of catalysts is summarised in Table 1.

XRD patterns of the HP catalysts indicated the presence of only anatase phase up to boiling times of $2 \mathrm{~h}$. For boiling times of 4 and $6 \mathrm{~h}$ the rutile phase also appears being the anatase the predominant one; and at $8 \mathrm{~h}$ almost only rutile is present. The crystallite sizes, as obtained from Scherrer's equation, were in the 5-9 $\mathrm{nm}$ range. In the literature $[32,33]$ preparation of rutile is generally carried out at very high temperature (ca. $1000 \mathrm{~K}$ ) but in very acidic medium anatase phase may transform to rutile at low temperature [34-36] giving rise to anatase-rutile mixtures whose ratio depends on the treatment duration.

BET specific surface areas were found to be ca. $220 \mathrm{~m}^{2} \cdot \mathrm{g}^{-1}$ for all the HP catalysts except for HP8 which exhibited a much lower value $\left(108 \mathrm{~m}^{2} \mathrm{~g}^{-1}\right)$, probably due to the anatase to rutile transformation occurring for this long boiling time. All the values are however considerably higher than those of commercial $\mathrm{TiO}_{2}$ samples used due to the lesser crystallinity of HP catalysts. 
The HP samples showed to be mesoporous and the total porosity decreased with boiling time. Figure 1 reports SEM images of the HP0.5 and HP8 samples showing that the catalysts consist of agglomerates. The average diameter of the agglomerates was ca. $25 \mathrm{~nm}$ and it was independent of boiling time.

\subsection{Photoreactivity}

Preliminary oxidation runs of BA and MBA were carried out in the 3-11 pH range and with different amounts of catalyst, being the optimality criterion that of maximising the selectivity. The highest selectivity to aldehyde was observed at $\mathrm{pH}=7$. Even if the oxidation rates increase with the catalyst amount, the highest selectivity was reached for HP catalysts with amounts of $0.4 \mathrm{~g} \cdot \mathrm{L}^{-1}$ for BA and of $0.2 \mathrm{~g} \cdot \mathrm{L}^{-1}$ for MBA. All the photoreactivity runs were therefore carried out with those catalyst amounts and at neutral $\mathrm{pH}$. For commercial catalysts the reaction rate increased and the selectivity sharply decreased at increasing catalyst amount; on this basis it was decided to use a powder amount of $0.02 \mathrm{~g} \cdot \mathrm{L}^{-1}$ for both aromatic alcohols as for this amount there was a high selectivity at a measurable reaction rate.

The main intermediate products detected in the course of BA and MBA photocatalytic oxidation were the corresponding aldehydes, $\mathrm{CO}_{2}$ being the other oxidation product. For $\mathrm{BA}$ in the presence of commercial $\mathrm{TiO}_{2}$ benzoic acid, hydroxylated derivatives and aliphatic compounds were observed while in the presence of HP catalysts the previous compounds were present in traces being hydroxylated derivatives absent. For MBA all the used catalysts did not produce any other intermediates except 4-methoxybenzoic acid that was detectable only for long lasting runs when the conversions were higher than $80 \%$. All the reactivity runs indicated that the rates of formation of aromatic aldehydes and $\mathrm{CO}_{2}$ had values different from zero from the starting of irradiation thus suggesting that their formation started contemporarily once the irradiation was turned on.

For all the used catalysts Table 2 reports the values of the irradiation time, $t_{\text {irr. }}$, needed for achieving a BA conversion of $50 \%$ together with the corresponding values of overall selectivity to aldehyde; these values are relative to $t_{\text {irr. }}$ and have been calculated as the ratio between the produced moles of aldehyde and the reacted moles of aromatic alcohol. During irradiation indeed the overall and the instantaneous selectivities had almost the same value. For the sake of comparison Table 2 also reports the data obtained for MBA oxidation [24] at a $65 \%$ conversion. 
The previous values of conversion were chosen as for them the aldehyde concentration reached the optimum value.

For HP0.5, the most selective photocatalyst, a specific study has been carried out to determine the influence of aliphatic alcohols (methanol, ethanol, 2-propanol, and tert-butanol) on reaction rate and selectivity. Preliminary photocatalytic runs were carried out with each aliphatic alcohol in the absence of aromatic alcohol and indicated that the HP0.5 was able to oxidize them. Figure 2 reports the concentration values of methanol versus the irradiation time for representative runs carried out without aromatic alcohol and in the presence of MBA. In this case the typical intermediates of methanol oxidation, such as formaldehyde and formic acid, were not detected in the slurries of HP0.5 catalyst and $\mathrm{CO}_{2}$ was the only product detected [37]. This finding has been also confirmed by the fact that carbon balance was satisfied in the course of the runs by only methanol and $\mathrm{CO}_{2}$. On the contrary, the photocatalytic degradation of ethanol, 2propanol, and tert-butanol produced a lot of stable intermediate compounds, that eventually were mineralized. On this ground all the data obtained in the course of BA and MBA degradation runs in the presence of methanol are representative of the methanol influence on the degradation process. For the runs carried out with the other aliphatic alcohols, only the data at low aliphatic alcohol conversion should be taken into account since at high conversion also the stable intermediates deriving from aliphatic alcohol degradation may affect the process.

The reactivity results obtained from representative runs of BA and MBA oxidation in the presence of methanol are reported in Figs. 3 and 4, respectively. From these Figures it may be noted that the data of aromatic aldehyde and $\mathrm{CO}_{2}$ production show a slope different from zero from the starting of irradiation thus suggesting that aldehyde and $\mathrm{CO}_{2}$ are contemporarily produced, as also observed for runs without alcohol. HPLC analyses revealed that the same chemical intermediates were formed from the aromatic alcohols also in the presence of methanol. Moreover, no volatile organic compounds were detected in the gaseous stream leaving the photoreactor, except negligible amounts of methanol desorbed from the aqueous solution. In Figs. 3 and 4, to clearly illustrate the contribution of different species to the carbon balance, the species concentrations have been normalized to carbon, thus multiplying the concentration of BA, BAD and benzoic acid by 7 (Figure 3) and that of MBA and PAA by 8 (Figure 4). Since no intermediates of methanol oxidation were detected, it was assumed that the entire reacted methanol was transformed into $\mathrm{CO}_{2}$. The total amount of $\mathrm{CO}_{2}$, produced by the aliphatic and 
aromatic alcohol mineralization, was calculated as the difference between the starting carbon content and the TOC measurement. The carbon balance, performed by summing the carbon amounts of aromatic alcohol, aliphatic alcohol, aldehyde, aromatic acid and $\mathrm{CO}_{2}$, was satisfactorily attained for all the runs, as Figures 3 and 4 show. In these Figures the continuous lines show the BA and MBA decrease with irradiation time for runs carried out without aliphatic alcohol; it may be noted that the oxidation of BA and MBA proceeds faster in the absence of aliphatic alcohol. The aromatic alcohol plays the same role on the oxidation rate of the aliphatic one; the data reported in Fig. 2 show that the methanol degradation rate in the presence of MBA is quite lower than in its absence.

Table 3 reports the selectivity and $t_{\text {irr }}$ values for BA and MBA oxidation in the presence of HP0.5 at different initial concentrations of methanol, ethanol, 2-propanol and tert-butanol. These values refer to a BA conversion of $50 \%$ and a MBA conversion of $65 \%$. This Table also reports selectivity and $t_{\text {irr }}$ values obtained for BA oxidation in the presence of ethanol with Degussa P25. For some representative runs Figures 5 and 6 report the concentration values of BAD and PAA, respectively, versus the irradiation time for runs carried out at increasing initial concentrations of aliphatic alcohols. It must be outlined that the PAA data of Fig. 6 are the initial ones, i.e. those for which the stable intermediates deriving from 2-propanol degradation do not affect the MBA oxidation process.

\section{DISCUSSION}

All the reactivity results indicate that the aromatic alcohol disappearance occurs through two parallel reactions: partial oxidation to the corresponding aldehyde and total oxidation to $\mathrm{CO}_{2}$. The occurrence of two parallel pathways for BA and MBA suggests that the aromatic alcohol molecules may interact with the $\mathrm{TiO}_{2}$ surface in two different ways that eventually determine their partial oxidation or mineralization. This is in agreement with Palmisano et al. [38] who have recently modeled the oxidation kinetics of aromatic molecules in water onto $\mathrm{TiO}_{2}$ surface by invoking the existence of two types of sites which are specific for the occurrence of mineralization or partial oxidation. In the mineralizing sites the aromatic alcohols molecules adsorb and produce $\mathrm{CO}_{2}$; it is likely that the mineralisation does not occur in a single step but it 
proceeds through a series of intermediates that do not desorb to the bulk of solution. In the partially oxidizing sites they adsorb and produce the aldehyde stable intermediates able to desorb to the bulk of solution. Once in the solution the fate of aldehyde molecules is the same of alcohol ones.

The structural results reported in Table 1 indicate that the cristallinity increases with the boiling time which also favours the crystal phase change from anatase to rutile. Reactivity results reported in Table 2 indicate that both for BA and MBA oxidation carried out with HP catalysts the reaction rates increase by increasing the cristallinity being the contemporary presence of anatase and rutile favourable, as also shown by $\mathrm{P} 25 \mathrm{TiO}_{2}$ which exhibits the highest reactivity even if at very low amount. Selectivities decrease with increasing cristallinity except for HP8, probably due to the predominant presence of the rutile structure [24]. HP0.5 exhibits the lowest reaction rate among the HP catalysts, but the highest selectivity for both aldehydes among all the used catalysts. The irradiation times needed to reach the selected BA and MBA conversions are similar each other but higher than those showed by Degussa P25. It is worth reporting that modifying irradiation time and/or decreasing catalyst amount of commercial samples did not result in selectivities comparable with that of HP0.5.

The attained selectivities point to a quite different behaviour of the two aromatic alcohols. The selectivity of BA to BAD is quite lower than that of MBA to PAA. A likely explanation is that the MBA's methoxy group (an electron donating one) increases the hydrophilicity of aromatic ring; in other words it increases the desorption capacity from the $\mathrm{TiO}_{2}$ surface [38] thus reducing the possibility of direct mineralization to $\mathrm{CO}_{2}$. It was recently reported for hydroxylation reactions by heterogeneous photocatalysis $[38,39]$ that the partial oxidation route is preferred when the aromatic ring contains an electron donor group whereas mineralization is favoured by an electron withdrawing substituent. This general rule, verified for many compounds in hydroxylation reactions, seems to hold also in the partial oxidation of an aromatic alcohol to aldehyde in water.

As to concern the influence of aliphatic alcohol, from the data reported in Table 3 it is evident that methanol and ethanol determine the highest increase in the selectivity values, being this positive effect directly linked to the aliphatic alcohol concentration. The selectivity improvement is higher for MBA than BA. It can be seen that in the case of MBA a selectivity improvement by 1.5 -fold is reached with methanol concentration of $38 \mathrm{mM}$ while for BA the 
selectivity increase is 1.25 -fold at the same methanol concentration. These findings suggest that the aliphatic alcohol can more successfully compete with MBA for the mineralizing pathway than with BA. This could be again ascribed to the presence of an electron donor group in MBA, responsible of supporting the partial oxidation route instead of mineralization. The $t_{\text {irr }}$ values obtained in the presence of aliphatic alcohols are all higher than those obtained without them, thus indicating that the alcohols and their stable intermediates negatively affect the overall oxidation rate of aromatic alcohol.

The increasingly better performance of aliphatic alcohols with the decrease of molecular weight can be probably ascribed to a more significant extent of photoadsorption for methanol and ethanol, due to a low steric hindrance. This effect is not evident for the highest aliphatic alcohol concentration used in this work, owing to a saturation effect of $\mathrm{TiO}_{2}$ surface.

The values of BAD concentration (see Fig. 5) do not change by the addition of $1 \mathrm{mM}$ methanol thus indicating that the production rate of BAD is not affected by the aliphatic alcohol at this concentration. At higher methanol concentrations the concentration values of BAD decrease and as a consequence also its production rate. The same qualitative behaviour is observed for the PAA concentration values with the difference that they were insensitive to alcohol concentration until a $10 \mathrm{mM}$ value (see Fig. 6). At high concentrations of aliphatic alcohol, even if the selectivity to aldehydes shows a small increase (see Table 3), the aldehyde production rate appreciably decreases (see Figs. 5 and 6) thus suggesting that for high concentrations the aliphatic alcohol also competes for the partially oxidizing pathway. These findings indicate not only that the interaction between the catalyst surface and the two aromatic substrates is different but also that there is a probable stronger competition of the aliphatic alcohol for the partially oxidizing pathway in the case of BA oxidation. BA was more easily mineralized than MBA in the presence of HP0.5 and it could interact less with the catalyst surface where partial oxidation occurs, so that for BA this pathway worsens in favour of the aliphatic alcohol.

In conclusion aliphatic alcohols appreciably raise the aldehyde selectivity owing to the fact that these compounds, being highly reducing species, favourably compete with the aromatic alcohols for the mineralizing pathway then lowering their overall disappearance rate. The occurrence of competition between aliphatic and aromatic alcohol molecules is also evidenced by the results reported in Fig. 2; in the presence of MBA methanol mineralization is about 2.5 times 
slower than in its absence. The favourable effect of aliphatic alcohol on selectivity seems to be specific of HP catalysts; in fact, when Degussa P25 was used with ethanol (see Table 3), the selectivity showed a negligible increase being also negligible the decrease of the overall oxidation rate. Likely explanations of the different behaviour of used catalysts are presented in an accompanying contribution [40].

In order to quantitatively characterize the influence of alcohol presence on the partial oxidation and mineralization processes, a rate equation has been fitted to the experimental data of $\mathrm{BA}, \mathrm{BAD}, \mathrm{MBA}$ and PAA concentrations. A first order kinetic equation, which has only a phenomenological meaning, satisfactorily $\left(\mathrm{R}^{2}>0.98\right)$ fits the data. The disappearance rate of aromatic alcohol $\left(-\mathrm{r}_{\mathrm{AA}}\right)$ and the appearance rates of aldehyde, $\mathrm{r}_{\mathrm{PO}}$, and carbon dioxide, $\mathrm{r}_{\mathrm{MIN}}$, are expressed as:

$$
\begin{aligned}
& \left(-\mathrm{r}_{\mathrm{AA}}\right)=-\frac{\mathrm{dC}_{\mathrm{AA}}}{\mathrm{dt}}=\left(\mathrm{k}_{\mathrm{PO}}+\mathrm{k}_{\mathrm{MIN}}\right) \mathrm{C}_{\mathrm{AA}} \\
& \mathrm{r}_{\mathrm{PO}}=\frac{\mathrm{dC}_{\mathrm{ALD}}}{\mathrm{dt}}=\mathrm{k}_{\mathrm{PO}} \mathrm{C}_{\mathrm{AA}} \\
& \mathrm{r}_{\mathrm{MIN}}=\frac{\mathrm{dC}_{\mathrm{CD}}}{\mathrm{dt}}=\mathrm{k}_{\mathrm{MIN}} \mathrm{C}_{\mathrm{AA}}
\end{aligned}
$$

in which $\mathrm{t}$ is the time, $\mathrm{k}_{\mathrm{PO}}$ and $\mathrm{k}_{\mathrm{MIN}}$ the first order rate constants for partial oxidation and mineralization, respectively, and $\mathrm{C}_{\mathrm{AA}}, \mathrm{C}_{\mathrm{ALD}}$, and $\mathrm{C}_{\mathrm{CD}}$ the aromatic alcohol, aromatic aldehyde and carbon dioxide concentrations. Integration of eqn. 1 produces an exponential relationship between $\mathrm{C}_{\mathrm{AA}}$ and $\mathrm{t}$ from which the value of $\left(\mathrm{k}_{\mathrm{PO}}+\mathrm{k}_{\mathrm{MIN}}\right)$ may be obtained by a least squares best fitting procedure. For determining the values of $\mathrm{k}_{\mathrm{PO}}$ and $\mathrm{k}_{\mathrm{MIN}}$, division of the members of eqn. 2 by the corresponding members of eqn. 3 yields:

$$
\frac{\mathrm{dC}_{\mathrm{ALD}}}{\mathrm{dC}_{\mathrm{CD}}}=\frac{\mathrm{k}_{\mathrm{PO}}}{\mathrm{k}_{\mathrm{MIN}}}
$$

Then by integration of eqn. 4 it is found that: 
$\mathrm{C}_{\mathrm{ALD}}=\frac{\mathrm{k}_{\mathrm{PO}}}{\mathrm{k}_{\mathrm{MIN}}} \mathrm{C}_{\mathrm{CD}}$

since $\mathrm{C}_{\mathrm{ALD}}=\mathrm{C}_{\mathrm{CD}}=0$ at $\mathrm{t}=0$.

Figure 7 shows the values of first order rate constants corresponding to partial oxidation and mineralization of aromatic alcohols, obtained by regression made with experimental points of the first 4 hours of irradiation. Increasing the concentration of methanol produces a decreasing of both the rate constants, but the effect is much more evident for the mineralization constant thus confirming that methanol preferentially competes with the aromatic alcohols for the mineralizing pathway.

\section{CONCLUSIONS}

The photoreactivity results indicate that the aromatic alcohol molecules participate to two parallel oxidative processes on the catalyst surface: the first one is the partial oxidation giving rise to the aldehyde and the second one is the complete oxidation producing eventually $\mathrm{CO}_{2}$. In the first pathway the adsorbed aromatic alcohol is transformed into the corresponding aldehyde which desorbs from the surface, while in the second one the substrate is mineralised through consecutive oxidation steps producing species which remain adsorbed onto the surface. The addition to the reacting system of an aliphatic alcohol (methanol, ethanol, 2-propanol or tertbutanol) determines a significant improvement of the photoprocess selectivity towards aldehyde. The aliphatic alcohol, as they are strong hole-traps, favourably compete with aromatic alcohols for the mineralizing pathway thus determining the increase of aldehyde selectivity.

The present investigation clearly indicates that a low-weight aliphatic alcohol such as methanol (or better the safe ethanol), added in small amounts in aqueous $\mathrm{TiO}_{2}$ suspensions, can relevantly improve the photoprocess selectivity towards partial oxidation without production of intermediates that could give rise to interferences in the subsequent separation steps of the products. To the best of our knowledge this is the first work reporting as the aliphatic alcohols are able to enhance the selectivity of heterogeneous photocatalytic oxidation processes in water, opening intriguing perspectives also for green synthetic aims. 


\section{REFERENCES}

1. J.E. Klijn, J.B.F.N. Engberts, Nature 435 (2005) 746.

2. C. Li, L. Chen, Chem. Soc. Rev. 35 (2006) 68.

3. S. Narayan, J. Muldoon, M.G. Finn, V.V. Fokin, H.C. Kolb, K. Barry Sharpless, Angew. Chem. Int. Ed. 44 (2005) 3275.

4. G.B. Jacobson, C.T. Lee, Jr., S.R.P. da Rocha, K.P. Johnston, J. Org. Chem. 64 (1999) 1207.

5. B. Das, J. Banerjee, G. Mahender, A. Majhi, Org. Lett. 6 (2004) 3349.

6. B.M. Trost, I. Fleming, S.V. Ley (Eds.), Comprehensive Organic Synthesis (Vol. 7), Pergamon Press, Oxford, 1991.

7. T. Mallat, A. Baiker, Chem. Rev. 104 (2004) 3037.

8. G.J. ten Brink, I.W.C.E. Arends, R.A. Sheldon, Science 287 (2000) 1636.

9. D.I. Enache, J.K. Edwards, P. Landon, B. Solsona-Espriu, A.F. Carley, A.A. Herzing, M. Watanabe, C.J. Kiely, D.W. Knight, G.J. Hutchings, Science 311 (2006) 362.

10. C. Donze, P. Korovchenko, P. Gallezot, M. Besson, Appl. Catal. B: Environ. 70 (2007) 621.

11. M. Salavati-Niasari, J. Mol. Cat. A: Chem. 245 (2006) 192.

12. A. Fujishima, T.N. Rao, D.A. Tryk, J. Photochem. Photobiol. C: Photochem. Rev. 1 (2000) 1 .

13. M. Addamo, V. Augugliaro, S. Coluccia, M.G. Faga, E. García-López, V. Loddo,, G. Marcì, G. Martra, L. Palmisano, J. Catal. 235 (2005) 209.

14. G. Mele, R. Del Sole, G. Vasapollo, G. Marcì, E. García-López, L. Palmisano, J. Coronado, M.D. Hernández-Alonso, C. Malitesta, M.R. Guascito, J. Phys. Chem. B 109 (2005) 12347.

15. V. Augugliaro, M. Litter, L. Palmisano, J. Soria, J. Photochem. Photobiol. C: Photochem. Rev. 7 (2006) 127.

16. S. Yurdakal, G. Palmisano, V. Loddo, V. Augugliaro, L. Palmisano, J. Am. Chem. Soc. 130 (2008) 1568.

17. A. Maldotti, A. Molinari, R. Amadelli, Chem. Rev. 102 (2002) 3811.

18. H. Yoshida, Curr. Opin. Solid State Mat. Sci. 7 (2003) 435. 
19. G. Palmisano, V. Augugliaro, M. Pagliaro, L. Palmisano, Chem. Commun. (2007) 3425.

20. M.A. Gonzalez, S.G. Howell, S.K. Sikdar, J. Catal. 183 (1999) 159.

21. T. Caronna, C. Gambarotti, L. Palmisano, C. Punta, F. Recupero, Chem. Commun. 18 (2003) 2350 .

22. U.R. Pillai, E. Sahle-Demessie, J. Catal. 211 (2002) 434.

23. O.S. Mohamed, A.E.M. Gaber, A.A. Abdel-Wahab, J. Photochem. Photobiol. A: Chem. 148 (2002) 205.

24. G. Palmisano, S. Yurdakal, V. Augugliaro, V. Loddo, L. Palmisano, Adv. Synth. Catal. 349 (2007) 964.

25. K.-I. Shimizu, T. Kaneko, T. Fujishima, T. Kodama, H. Yoshida, Y. Kitayama, Appl. Catal. A: Gen. 225 (2002) 185.

26. R.I. Bickley, R.K.M. Jayanty, Faraday Discuss. Chem. Soc. 58 (1974) 194.

27. Y. Tamaki, A. Furube, M. Murai, K. Hara, R. Katoh, M. Tachiya, J. Am. Chem. Soc. 128 (2006) 416.

28. K.M. Schindler, M. Kunst, J. Phys. Chem. 94 (1990) 8222.

29. V. Brezová, P. Tarábek, D. Dvoranová, A. Staško, S. Biskupič, J. Photochem. Photobiol. A: Chem. 155 (2003) 179.

30. H. Yamashita, K. Ikeue, T. Takewaki, M. Anpo, Top. Catal. 18 (2002) 95.

31. G. Palmisano, M. Addamo, V. Augugliaro, T. Caronna, A. Di Paola, E. García-López, V. Loddo, G. Marcì, L. Palmisano, M. Schiavello, Catal. Today 122 (2007), 118.

32. A. Sclafani, L. Palmisano, M. Schiavello, J. Phys. Chem. 94 (1990) 829.

33 M. Addamo, V. Augugliaro, A. Di Paola, E. García-López, V. Loddo, G. Marci, R. Molinari, L. Palmisano, M. Schiavello, J. Phys. Chem. B 108 (2004) 3303.

34. M. Nag, P. Basak, S. V. Manorama, Mater. Research Bull. 42 (2007) 1691.

35. L. Li, J. Liu, Z. Jia, Mater. Lett. 60 (2006) 1753.

36. S. Yurdakal, G. Palmisano, V. Loddo, V. Augugliaro, L. Palmisano, J. Am. Chem. Soc. $130(2008) 1568$.

37. J. Chen, D. F. Ollis, W. H. Rulkens, H. Bruning. Water Res. 33, 1999, 661.

38. G. Palmisano, V. Loddo, S. Yurdakal, V. Augugliaro, L. Palmisano, AIChE J. 53 (2007) 961. 
39. G. Palmisano, M. Addamo, V. Augugliaro, T. Caronna, E. García-López, V. Loddo, L. Palmisano, Chem. Commun. (2006) 1012.

40. V. Augugliaro, H. Kisch, V. Loddo, M.J. López-Muñoz, C. Márquez-Álvarez, G.

Palmisano, L. Palmisano, F. Parrino, S. Yurdakal, Appl. Catal. A: Gen. (2008) submitted. 


\section{CAPTIONS}

Figure 1. SEM images (magnification $\times 200000$ ) of (a) HP0.5 and (b) HP8.

Figure 2. Concentration of methanol in the presence or absence of MBA $1 \mathrm{mM}$.

Figure 3. BA photocatalytic oxidation to BAD in the presence of $10 \mathrm{mM}$ methanol. The continuous line refers to BA concentration values obtained in a run without methanol. The concentrations are referred to carbon.

Figure 4. MBA photocatalytic oxidation to PAA in the presence of $10 \mathrm{mM}$ methanol. The continuous line refers to MBA concentration values obtained in a run without methanol. The concentrations are referred to carbon.

Figure 5. Concentration of BAD versus irradiation time in the presence of different initial concentrations of methanol. Initial BA concentration: $1 \mathrm{mM}$.

Figure 6. Concentration of PAA versus irradiation time in the presence of different initial concentrations of 2-propanol. Initial MBA concentration: $1 \mathrm{mM}$.

Figure 7. First order rate constants of mineralization and partial oxidation reactions of BA and MBA in the presence of different initial concentrations of methanol. Initial concentration of BA and MBA: $1 \mathrm{mM}$.

Table 1. Photocatalysts textural properties.

Table 2. Irradiation time and selectivity for aromatic alcohols oxidation to aldehydes.

Table 3. Selectivity values and irradiation times for BA and MBA in the presence of aliphatic alcohols at different initial concentrations. 


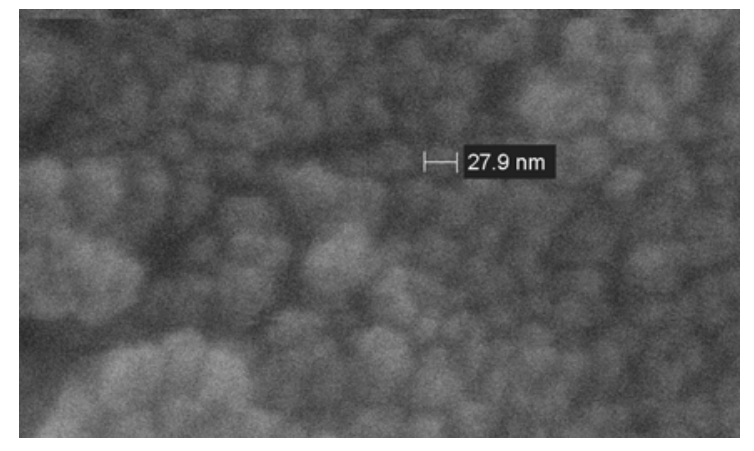

(a)

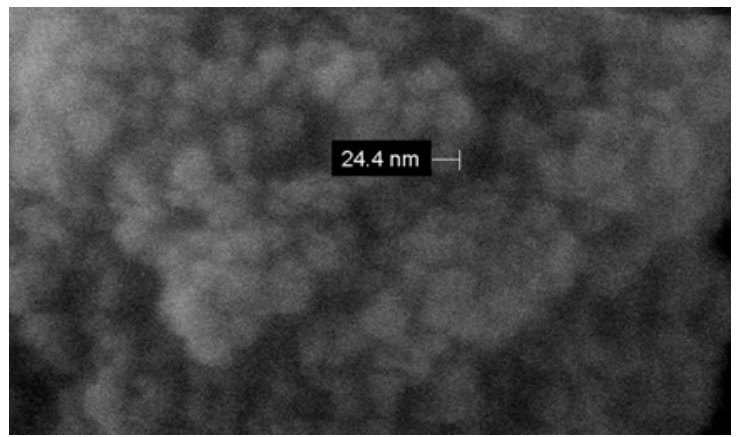

(b)

Figure 1. 


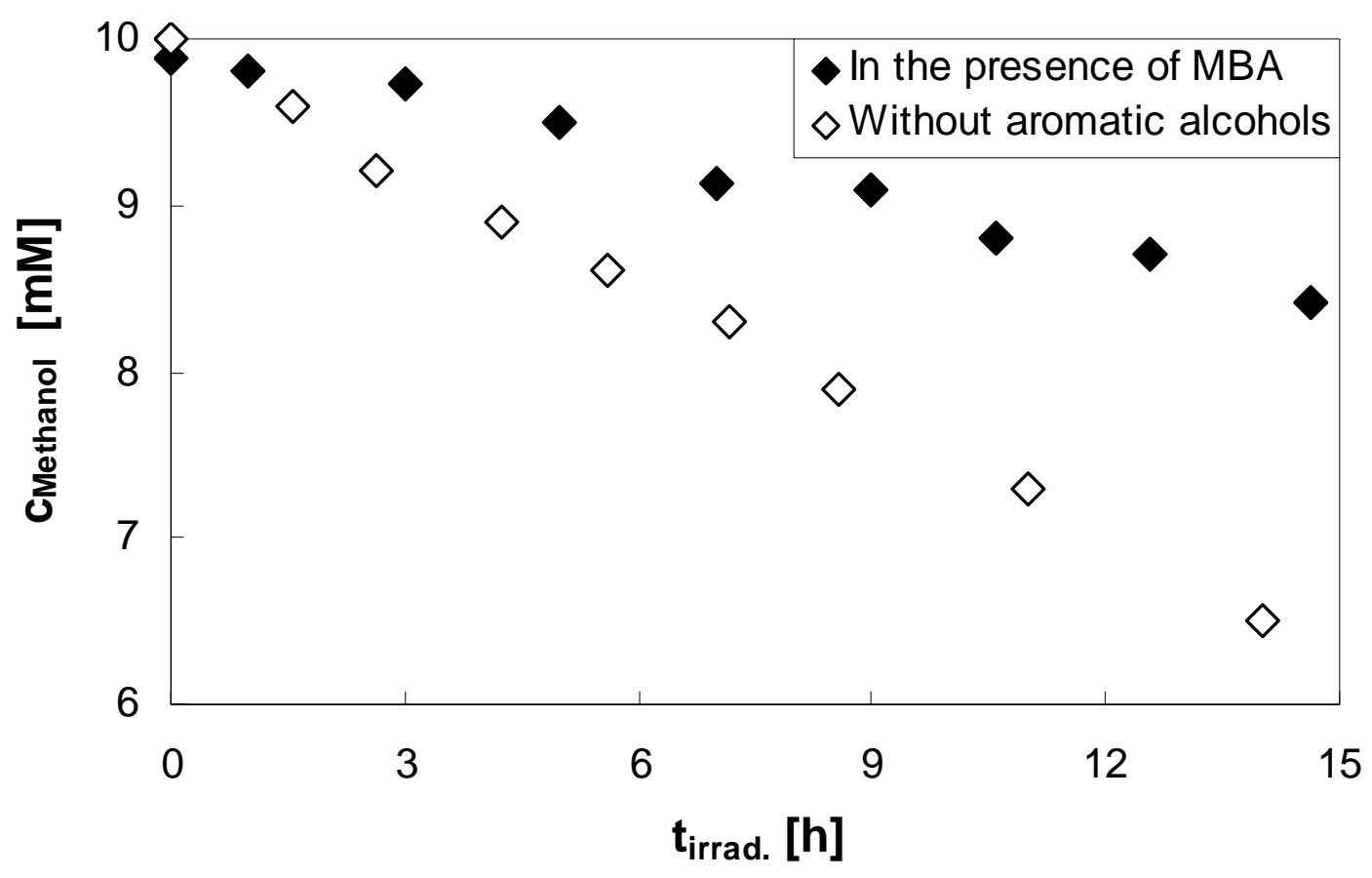

Figure 2 


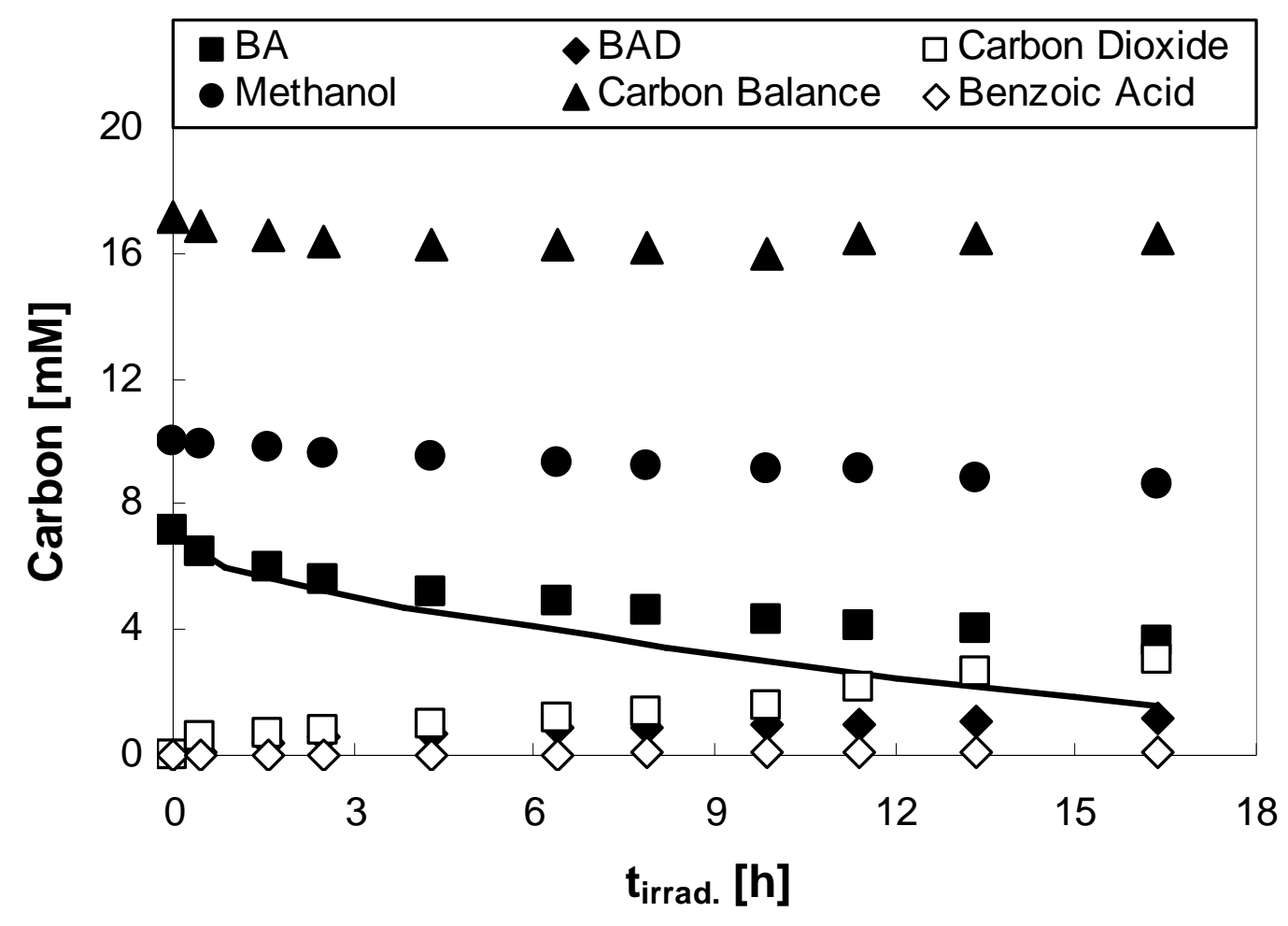

Figure 3 


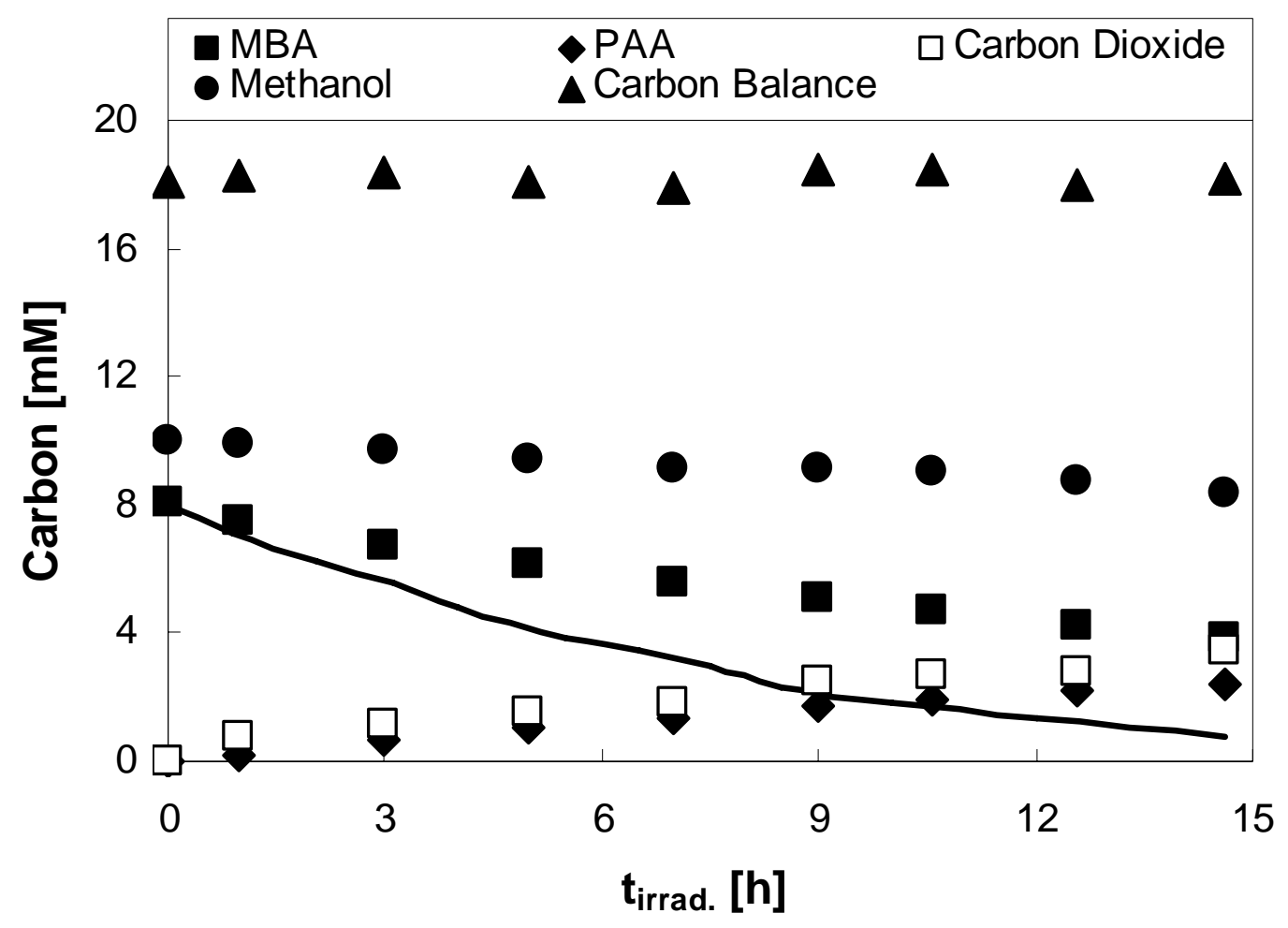

Figure 4 


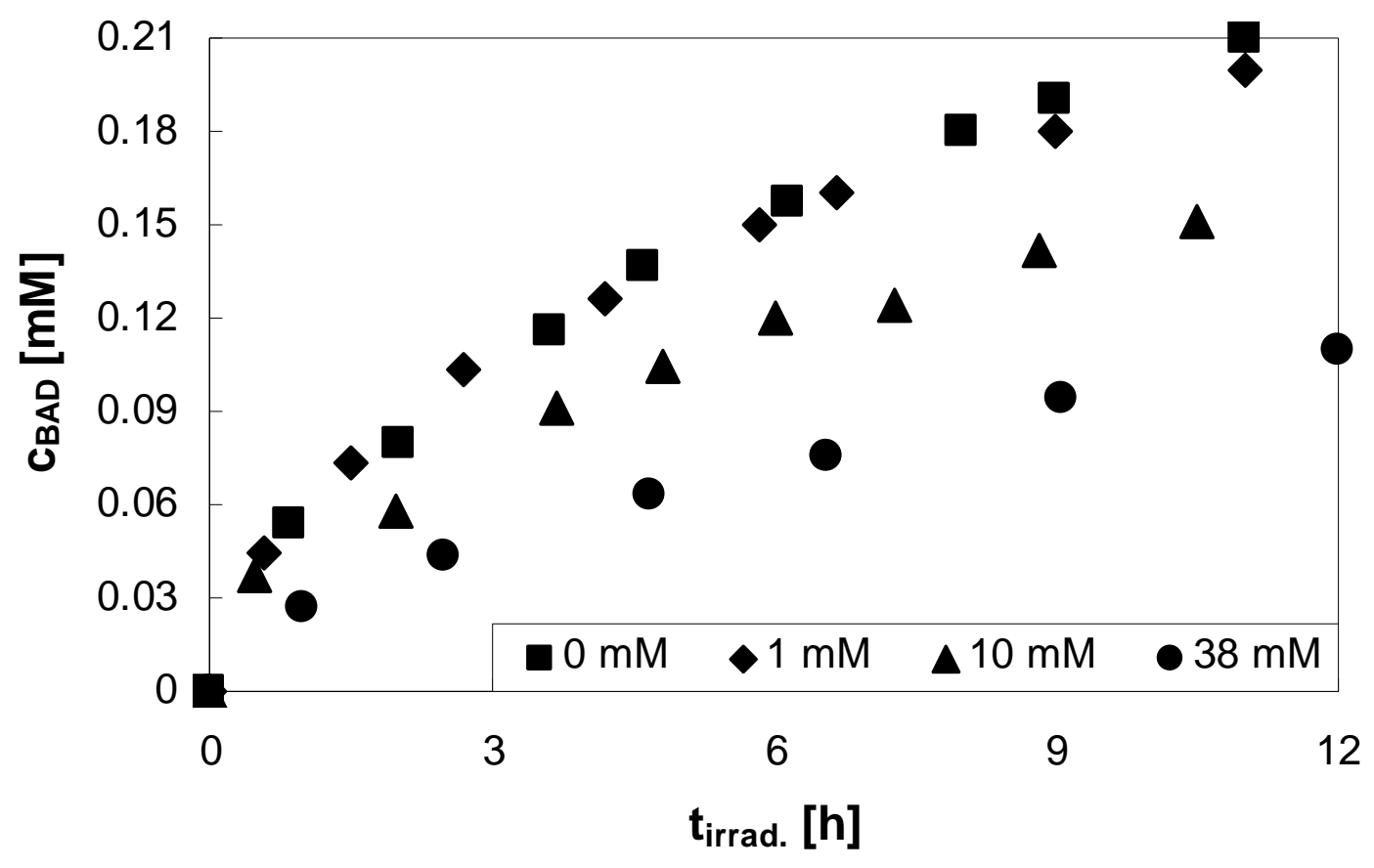

Figure 5 


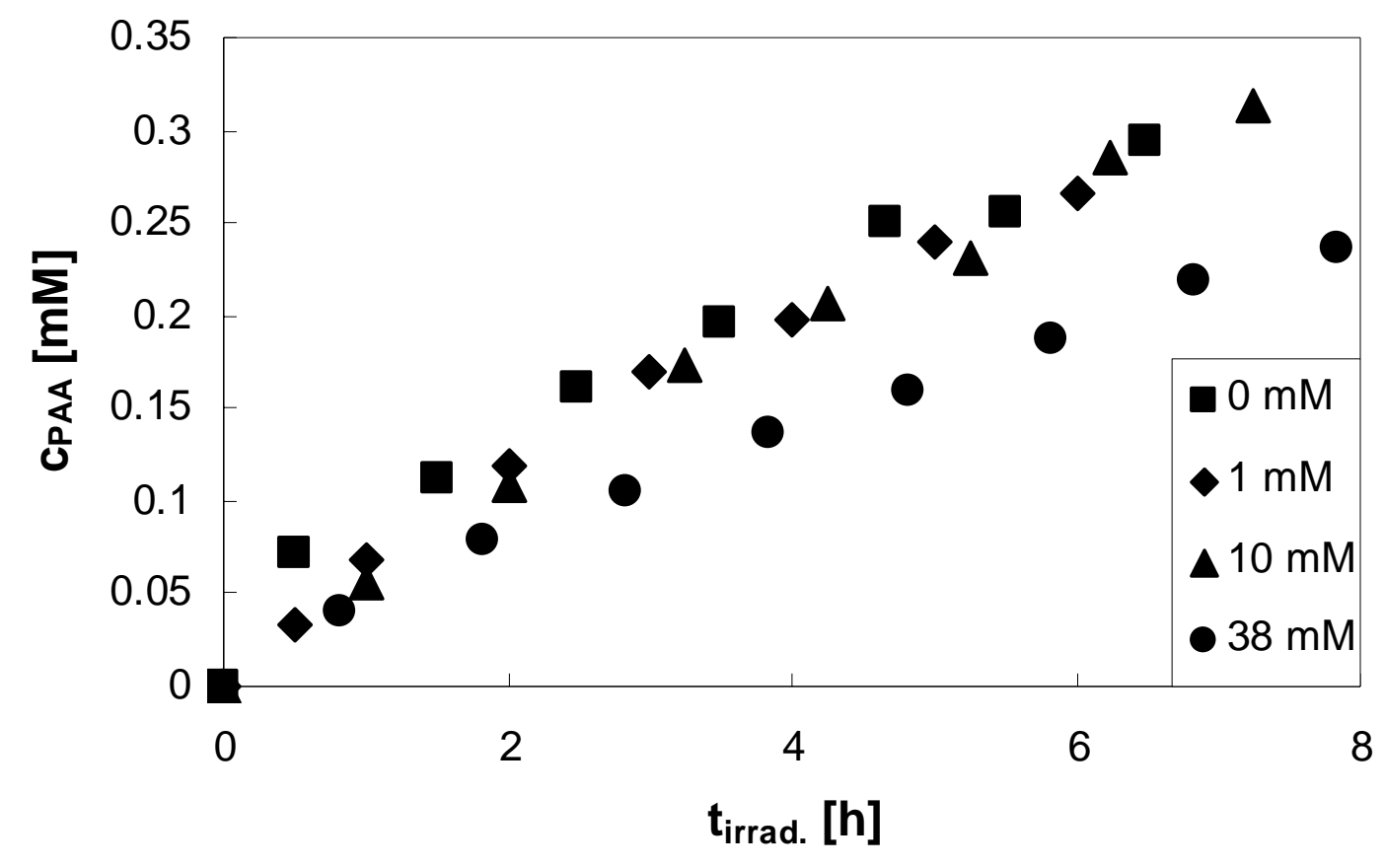

Figure 6 


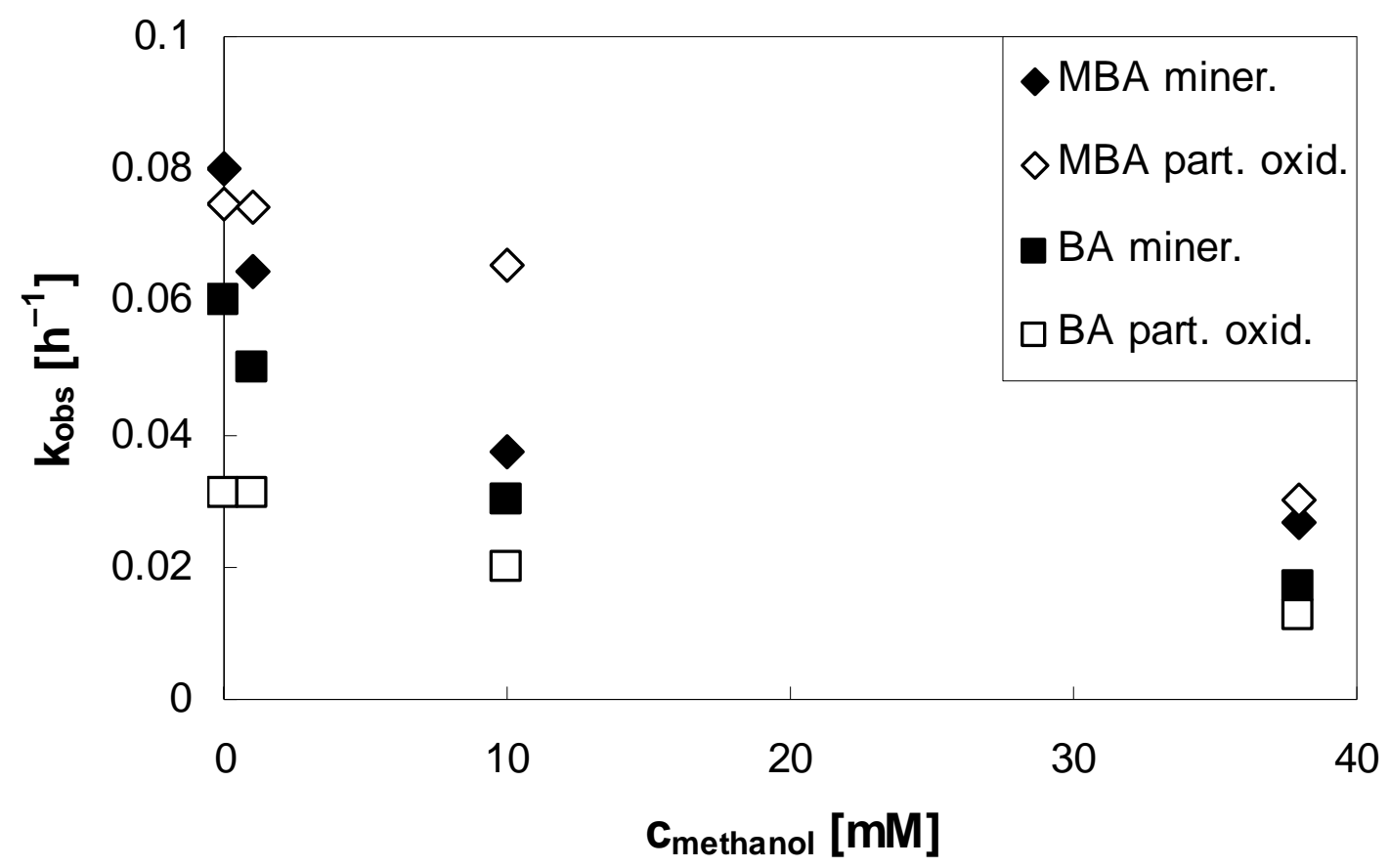

Figure 7 
Table 1.

\begin{tabular}{ccccc}
\hline Catalyst & $\begin{array}{c}\text { Phase } \\
\text { A, anatase } \\
\text { R, rutile }\end{array}$ & SSA & $\begin{array}{c}\text { Total } \\
\text { porosity } \\
{\left[\mathrm{m}^{2} \mathrm{~g}^{-1}\right]} \\
{\left[\mathrm{mm}^{3} \mathrm{~g}^{-1}\right]}\end{array}$ & $\begin{array}{c}\text { Average } \\
\text { pore radius } \\
{[\AA]}\end{array}$ \\
\hline Degussa P25 & $\mathrm{A}, \mathrm{R}$ & 50 & - & - \\
Merck & $\mathrm{A}$ & 10 & - & - \\
HP0.5 & $\mathrm{A}$ & 235 & 351 & 26 \\
HP2 & $\mathrm{A}$ & 226 & 325 & 29 \\
HP4 & $\mathrm{A}, \mathrm{R}$ & 220 & 318 & 28 \\
HP6 & $\mathrm{A}, \mathrm{R}$ & 206 & 292 & 28 \\
HP8 & $\mathrm{R}$ & 108 & 205 & 38 \\
\hline
\end{tabular}


Table 2.

\begin{tabular}{ccccccc}
\hline \multirow{3}{*}{ Photocatalyst } & \multicolumn{3}{c}{ Benzyl Alcohol } & \multicolumn{3}{c}{ 4-Methoxybenzyl Alcohol } \\
\cline { 2 - 7 } & $\begin{array}{c}\text { Catalyst } \\
\text { amount } \\
{\left[\mathrm{g} \mathrm{L}^{-1}\right]}\end{array}$ & $\begin{array}{c}t_{\text {irr. }} \\
{[\mathrm{h}]}\end{array}$ & $\begin{array}{c}\text { Selectivity } \\
{[\% \text { mol] }}\end{array}$ & $\begin{array}{c}\text { Catalyst } \\
\text { amount } \\
{\left[\mathrm{L} \mathrm{L}^{-1}\right]}\end{array}$ & $\begin{array}{c}t_{\text {irr. }} \\
{[\mathrm{h}]}\end{array}$ & $\begin{array}{c}\text { Selectivity } \\
{[\% \text { mol] }}\end{array}$ \\
\hline Degussa P25 & 0.02 & 3.9 & 8 & 0.02 & 5.6 & 11 \\
Merck & 0.02 & 17.6 & 9 & 0.02 & 13.8 & 14 \\
HP0.5 & 0.4 & 6.1 & 28 & 0.2 & 7.7 & 41 \\
HP2 & 0.4 & 6.0 & 26 & 0.2 & 4.7 & 37 \\
HP4 & 0.4 & 5.7 & 26 & 0.2 & 3.9 & 36 \\
HP6 & 0.4 & 4.3 & 24 & 0.2 & 2.8 & 31 \\
HP8 & 0.4 & 5.3 & 17 & 0.2 & 3.2 & 32 \\
\hline
\end{tabular}

$\mathrm{t}_{\text {irr. }}$ and selectivity refer to a BA and MBA conversion of $50 \%$ and $65 \%$, respectively. Selectivity is determined as (moles of aldehyde) / (moles of alcohol converted). Alcohol initial concentration: $1 \mathrm{mM}$. 
Table 3.

\begin{tabular}{|c|c|c|c|c|c|c|c|}
\hline & \multicolumn{3}{|c|}{$B A$} & \multicolumn{3}{|c|}{$M B A$} \\
\hline & & $\begin{array}{c}\text { Selectivity } \\
\text { for } 10 \% \\
\text { conversion } \\
\text { [\% mol] }\end{array}$ & $\begin{array}{c}\text { Selectivity } \\
\text { for } 50 \% \\
\text { conversion } \\
{[\% \text { mol] }}\end{array}$ & $\begin{array}{c}t_{i r r} \\
\text { for } 50 \% \\
\text { conversion } \\
{[\mathrm{h}]}\end{array}$ & $\begin{array}{c}\text { Selectivity } \\
\text { for } 10 \% \\
\text { conversion } \\
{[\% \mathrm{~mol}]}\end{array}$ & $\begin{array}{c}\text { Selectivity } \\
\text { for } 65 \% \\
\text { conversion } \\
{[\% \mathrm{~mol}]}\end{array}$ & $\begin{array}{c}t_{i r r} \\
\text { for } 65 \% \\
\text { conversion } \\
{[h]}\end{array}$ \\
\hline \multirow{2}{*}{\multicolumn{2}{|c|}{$\begin{array}{c}\text { Without aliphatic } \\
\text { alcohol }\end{array}$}} & 30 & 28 & 7.7 & \multirow[b]{2}{*}{44} & \multirow[b]{2}{*}{41} & \multirow[b]{2}{*}{7.7} \\
\hline & & $9^{*}$ & $8^{*}$ & $3.9^{*}$ & & & \\
\hline \multirow[t]{3}{*}{ Methanol } & $1 \mathrm{mM}$ & 34 & 30 & 8.2 & 55 & 50 & 9.0 \\
\hline & $10 \mathrm{mM}$ & 40 & 34 & 16.3 & 64 & 58 & 13.5 \\
\hline & $38 \mathrm{mM}$ & 43 & 38 & 23.7 & 68 & 62 & 17.2 \\
\hline \multirow[t]{4}{*}{ Ethanol } & $1 \mathrm{mM}$ & 36 & 30 & 8.0 & 60 & 54 & 8.9 \\
\hline & $10 \mathrm{mM}$ & 45 & 37 & 14.2 & \multirow{2}{*}{58} & \multirow{2}{*}{56} & \multirow{2}{*}{9.4} \\
\hline & & $10^{*}$ & $9^{*}$ & $4.1^{*}$ & & & \\
\hline & $38 \mathrm{mM}$ & 52 & 42 & 21.0 & 62 & 61 & 14.3 \\
\hline \multirow[t]{3}{*}{ 2-Propanol } & $1 \mathrm{mM}$ & 32 & 30 & 8.3 & 55 & 47 & 8.0 \\
\hline & $10 \mathrm{mM}$ & 40 & 34 & 12.3 & 57 & 52 & 10.2 \\
\hline & $38 \mathrm{mM}$ & 44 & 36 & 24.2 & 61 & 60 & 17.8 \\
\hline \multirow[t]{3}{*}{ Tert-butanol } & $1 \mathrm{mM}$ & 31 & 29 & 9.1 & 59 & 51 & 9.9 \\
\hline & $10 \mathrm{mM}$ & 41 & 32 & 11.9 & 56 & 54 & 10.5 \\
\hline & $38 \mathrm{mM}$ & 38 & 34 & 16.8 & 64 & 58 & 12.2 \\
\hline
\end{tabular}

Selectivity is determined as (moles of aldehyde)/(moles of alcohol converted). Initial concentration of aromatic alcohol: $1 \mathrm{mM}$. Catalyst: HP0.5, amount: $0.2 \mathrm{~g} \mathrm{~L}^{-1}$. The data with $\left(^{*}\right)$ have been obtained with Degussa P25, $0.02 \mathrm{~g} \mathrm{~L}^{-1}$. 